\title{
Le portage asymptomatique des Pestivirus chez les ruminants
}

\author{
P.-P. PASTORET, D. BOULANGER, B. MIGNON et S. WAXWEILER *
}

\begin{abstract}
Résumé : Les Pestivirus sont des virus enveloppés à acide ribonucléique monocaténaire de polarité positive. Ils regroupent le virus responsable de la peste porcine classique, le virus de la maladie de la frontière chez le mouton, celui responsable de la maladie des muqueuses chez les bovins, ainsi que des isolats obtenus chez des mammifères sauvages comme le cerf rouge (Cervus elaphus). Chez les ruminants, les Pestivirus ont développé une stratégie particulièrement remarquable pour assurer leur pérennité. Ils provoquent, par transmission épigénétique, la naissance d'animaux porteurs asymptomatiques de variants non cytopathogènes du virus et immunotolérants vis-à-vis de la souche de virus qu'ils hébergent.

La présence de porteurs asymptomatiques en faible nombre permet la circulation du virus, par transmission horizontale, à l'intérieur des troupeaux, assurant ainsi la naissance d'une nouvelle génération de porteurs asymptomatiques.
\end{abstract}

MOTS-CLÉS : Cervus elaphus - Epidémiologie - Immunotolérance Maladies virales - Pestivirus - Portage - Ruminants.

\section{INTRODUCTION}

L'infection asymptomatique des ruminants par les Pestivirus présente un intérêt particulier puisqu'elle est associée à un état d'immunotolérance $(13,31)$. Pendant longtemps, les connaissances sur les Pestivirus étaient difficiles à acquérir, du fait des propriétés particulières de ces virus qui rendent ardues leur production et leùr purification.

Depuis le premier séquençage du génome d'un Pestivirus par Renard et coll. $(34,35)$, les progrès réalisés dans la connaissance de la biologie moléculaire de ces virus ont été extrêmement rapides. Ces progrès ont également eu des répercussions sur la compréhension de la pathogénie des maladies associées à l'infection par les Pestivirus. Depuis que Brownlie et coll. ont, en 1984 (6), à la suite des travaux de l'équipe de Liess (22), reproduit expérimentalement la maladie des muqueuses, beaucoup d'inconnues sur les mécanismes responsables ont également été levées.

La clé de l'épidémiologie de ces infections est l'existence de porteurs asymptomatiques et immunotolérants de souches non cytopathogènes de virus, comme cela a été démontré chez les bovins et les moutons. L'existence d'états semblables chez

* Service de virologie-immunologie, Faculté de médecine vétérinaire, Université de Liège, Institut de chimie, Bât. B6, local R80, B-4000 Sart Tilman-Liège, Belgique. 
les ruminants sauvages comme le cerf́ rouge (Cervus elaphus), mériterait d'être recherchée afin de savoir s'il s'agit d'un phénomène biologique universellement adopté chez les ruminants.

\section{LES PESTIVIRUS}

Le genre Pestivirus regroupe actuellement trois virus identifiés depuis de nombreuses années : celui responsable de la peste porcine classique (hog cholera virus : $\mathrm{HCV}$ ) dont l'infection était déjà décrite dès le XIX ${ }^{\mathrm{e}}$ siècle, celui responsable de la diarrhée virale bovine (bovine virus diarrhoea virus: BVDV) et de la maladie des muqueuses, infections respectivement décrites par Olafson et coll. en 1946 (30) et Ramsey et Chivers en 1953 (33), et le virus de la maladie de la frontière (Border disease virus) chez le mouton, décrite quelques années plus tard (20). Il faut ajouter, à cès trois Pestivirus, certains autres virus isolés chez des ruminants sauvages comme le cerf rouge.

Des enquêtes sérologiques ont par ailleurs montré que ces infections devaient être répandues chez ces espèces $(15,21,29)$.

Deux biotypes du BVDV (cytopathogène et non cytopathogène) distinguables en culture de cellules, ont été isolés chez les bovins atteints de maladie des muqueuses, alors que les animaux infectés de manière persistante n'hébergent que le biotype non cytopathogène (6).

Les Pestivirus, virus enveloppés à acide ribonucléique (ARN) monocaténaire infectieux, de polarité positive, classés auparavant dans la famille des Togaviridae (40), s'en distinguent par l'absence d'ARN subgénomique et d'une queue polyadénylée à leur extrémité $3^{\prime}(32,34)$ ainsi que par leur organisation génomique (10). Ces différences fondamentales ont poussé le Comité international de taxonomie des virus à reclasser le genre Pestivirus dans la famille des Flaviviridae créée en 1985 (41) et qui, jusqu'à présent, ne comprenait qu'un seul genre viral, les Flavivirus (17).

Les génomes de deux souches de BVDV $(10,34)$ et de deux souches de HCV $(25,28)$ ont été clonés et séquencés. Chez ces deux espèces de virus, une seule phase de lecture ouverte couvrant pratiquement toute la longueur de l'ARN (12 à 13 kilobases) et pouvant coder pour environ 450 kilo-Daltons en protéines a été mise en évidence. Deux courtes phases de lecture ouvertes ont également été décrites chez la souche bovine NADL en amont de la longue phase de lecture ouverte, mais elles ne semblent pas être fonctionnelles $(10,42)$.

L'étude de l'organisation génomique du BVDV (11) suggère que, comme chez les Flavivirus, les protéines de structure sont codées par le premier tiers du génome à l'extrémité 5', tandis que les protéines non structurales sont codées par les deux tiers restants. La première de ces protéines non structurales, la p125, présente la particularité d'être clivée, uniquement chez le biotype cytopathogène, en p54 et p80. La protéine p54 contient une séquence riche en cystéines (Cys) similaire aux séquences des protéines «en doigt de zinc» pouvant interagir avec des acides nucléiques (14), tandis que la p80 posséderait une activité hélicase et protéolytique responsable du clivage des protéines non structurales (43). La comparaison de séquences de plusieurs souches de Pestivirus a mis en évidence la présence, chez certaines souches cytopathogènes de BVDV, d'insertions de gènes cellulaires localisés dans la région codant pour l'extrémité C terminale de la p54 et se traduisant par une modification du poids moléculaire de cette 
protéine et de ses précurseurs (1). Chez la souche NADL, la séquence insérée correspond au gène d'une protéine cellulaire non identifiée (26), tandis que dans le cas de la souche «OSc», l'insert code pour un monomère d'ubiquitine animale $(5,26)$.

Meyers et coll. (27) ont également décrit, chez une souche cytopathogène isolée d'un animal atteint de maladie des muqueuses, l'insertion d'une séquence correspondant à un monomère complet d'ubiquitine, un fragment d'un second monomère et une duplication d'une séquence virale. D'autres souches virales cytopathogènes ne semblent cependant pas contenir de telles insertions (14).

\section{RÉPARTITION DES SOUCHES CYTOPATHOGÈNES OU NON CYTOPATHOGÈNES CHEZ LES BOVINS INFECTÉS}

Clarke et coll. (9) ont examiné la répartition des souches, cytopathogènes ou non, dans les tissus d'animaux infectés de manière asymptomatique ou morts de la maladie des muqueuses. Cette recherche avait été entreprise à la suite de trois constatations:

- les bovins infectés de manière persistante par une souche non cytopathogène du BVDV peuvent succomber ultérieurement de maladie des muqueuses, le plus souvent à l'âge de 6 à 24 mois $(22,24,36,37)$;

- on retrouve chez les bovins atteints de maladie des muqueuses, les deux types de souches ;

- la maladie des muqueuses peut être expérimentalement reproduite par la surinfection d'animaux porteurs asymptomatiques d'un biotype non cytopathogène, à l'aide d'un biotype cytopathogène antigéniquement identique $(4,6)$.

Clarke et coll. (9) ont tout d'abord examiné 28 cas de maladie des muqueuses survenus naturellement et 53 animaux souffrant de virémie persistante qui ne présentaient aucun signe clinique. Des souches cytopathogènes et non cytopathogènes étaient concomitamment isolées chez les animaux qui souffraient de maladie des muqueuses, alors que seules des souches non cytopathogènes étaient isolées des animaux infectés de manière persistante. Ces observations, en plus des résultats obtenus par reproduction expérimentale de la maladie des muqueuses, confirment que les deux biotypes sont nécessaires pour provoquer cette maladie. Ces mêmes auteurs ont observé davantage de souches cytopathogènes dans les prélèvements intestinaux, alors que les souches non cytopathogènes étaient associées aux cellules sanguines, aux organes associés à la circulation sanguine et au tractus respiratoire. Ces résultats ont été confirmés par Bielefeldt Ohmann et coll. (2).

Les deux types de souches semblent donc manifester un tropisme différent. Des antigènes de souches non cytopathogènes ont également été démontrés au niveau du système nerveux central des animaux infectés de manière persistante (16).

\section{PATHOGÉNIE ET ÉPIDÉMIOLOGIE DE LINFECTION PAR LE VIRUS DE LA DIARRHÉE VIRALE BOVINE}

Le mode de transmission et le biotype (cytopathogène ou non cytopathogène) de la souche infectante influencent la pathogénie de l'infection. 
Lors d'une transmission horizontale, le virus, généralement non cytopathogène, pénètre au niveau oro-nasal, conjonctival ou génital, s'y multiplie, puis est transporté par voie sanguine vers d'autres organes cibles comme la muqueuse intestinale. Ce mode de transmission peut entraîner l'apparition d'une diarrhée ordinairement bénigne s'accompagnant d'une fièvre modérée et d'une leucopénie transitoire. Des anticorps neutralisants apparaissent deux à trois semaines après l'infection (7).

$\mathrm{Si}$, par contre, une vache est infectée en cours de gestation, le virus peut traverser la barrière placentaire et contaminer le foetus (transmission épigénétique). L'issue de l'infection dépendra du biotype et du stade du développement fœtal au moment où elle se produit. Si une souche non cytopathogène infecte une vache entre les $80^{\mathrm{e}}$ et $110^{\mathrm{e}}$ jours de gestation, il peut s'ensuivre la naissance de veaux infectés de manière persistante (23) par le biotype non cytopathogène de la souche infectante, et immunotolérants envers cette souche. L'immunotolérance est acquise par présentation des antigènes viraux pendant la période critique de l'acquisition de la tolérance (13).

Ces veaux, apparemment sains dans la majorité des cas, excrètent continuellement le biotype non cytopathogène du virus et contaminent le reste du troupeau de manière silencieuse (38).

Si le foetus est infecté à un stade plus avancé de la gestation, il développe une immunité spécifique active, et stérilisante.

Si le foetus est infecté au stade critique par un biotype cytopathogène, les conséquences de l'infection différent également (8). Le biotype cytopathogène peut également traverser la barrière placentaire, mais sans provoquer la naissance de veaux infectés de manière persistante. Brownlie et coll. (8) ne sont pas parvenus à réisoler ce biotype chez des foetus infectés six jours auparavant. Ils suggèrent dès lors que les cellules cibles du biotype cytopathogène, contrairement à celles du biotype non cytopathogène, ne sont pas encore différenciées avant la période d'immunocompétence, ce qui empêcherait le virus de s'établir de manière persistante.

Comme il est mentionné ci-dessus, seuls les veaux infectés de manière persistante peuvent développer la maladie des muqueuses à issue toujours fatale. Celle-ci surviendrait après surinfection du veau infecté de manière persistante par un biotype cytopathogène antigéniquement identique. Une surinfection par une souche antigéniquement hétérologue, induirait une śéroconversion d'autant plus importante que la souche surinfectante est antigéniquement plus éloignée de la souche non cytopathogène persistante (3). Ce phénomène permet d'expliquer la présence d'anticorps spécifiques chez des animaux infectés de manière persistante (38) qui devraient normalement en être dépourvus puisqu'ils sont immunotolérants.

\section{DÉCLENCHEMENT DE LA MALADIE DES MUQUEUSES}

Le déclenchement de la maladie des muqueuses est associé à l'apparition d'une souche cytopathogène antigéniquement identique à la souche non cytopathogène hébergée. Plusieurs hypothèses peuvent être proposées pour expliquer l'origine du biotype cytopathogène. Corapi et coll. (12) ont montré, à l'aide d'une gamme d'anticorps monoclonaux, que les deux biotypes d'une souche virale de BVDV isolée chez un animal atteint de maladie des muqueuses étaient antigéniquement semblables. Deux hypothèses ont alors été avancées. 
Selon la première, les souches cytopathogènes dériveraient des souches non cytopathogènes après mutation au niveau du site catalytique ou au niveau du site du clivage $(7,18)$. Une mutation au niveau du site catalytique est cependant peu probable, puisqu'il a été démontré que l'activité protéolytique de la protéine p80 est responsable du clivage de la plupart des protéines non structurales du BVDV (43) et qu'un seul de ces clivages est altéré chez les souches cytopathogènes.

La seconde hypothèse, impliquant un phénomène original de recombinaison entre des séquences nucléotidiques cellulaires et le génome des souches non cytopathogènes, se base sur la mise en évidence d'insertions de séquences nucléotidiques cellulaires dans le gène codant pour la p54. Ces insertions seraient responsables du clivage de la p125 (27).

A l'heure actuelle, de pareilles insertions ont été observées chez plusieurs souch $\backsim$ s cytopathogènes, mais leur absence chez d'autres souches (14) semble indiquer que cette hypothèse ne suffit pas à expliquer l'apparition des biotypes cytopathogènes.

Comme les deux biotypes du BVDV se distinguent uniquement par le clivage de la p125 chez le biotype cytopathogène, l'apparition de la cytopathogénicité pourrait être liée à une ou plusieurs propriétés des protéines issues du clivage, distinctes de celle(s) du précurseur.

Le caractère cytopathogène pourrait être dû à la présence du «domaine en doigt de zinc» au niveau de la p54, dont l'activité serait modifiée chez son précurseur en raison d'une conformation différente (14).

La cytopathogénicité pourrait au contraire résulter de l'activité protéolytique de la p80. Or, cette protéine présente vraisemblablement une distribution intracellulaire différente chez les deux biotypes. En effet, elle serait libre chez le biotype cytopathogène, alors que, étant liée à la p54 chez le biotype non cytopathogène, sous la forme de p125, elle possède une extrémité $\mathrm{N}$ terminale très hydrophobe qui l'associerait à une membrane, ainsi qu'une séquence «en doigt de zinc» qui l'associerait à des acides nucléiques (43).

\section{CIRCULATION DES SOUCHES NON CYTOPATHOGÈNES ET ÉPIDÉMIOLOGIE DE L'INFECTION}

Les veaux infectés de manière persistante jouent un rôle essentiel dans l'épidémiologie de l'infection par le BVDV, puisque ces animaux sont, en définitive, la source des biotypes non cytopathogènes et, après mutation ponctuelle ou recombinaison, également la source des biotypes cytopathogènes capables de déclencher la maladie des muqueuses. Les souches cytopathogènes qui émergent de la population de biotypes non cytopathogènes peuvent se transmettre aux porteurs persistants immunotolérants et les éliminer par la maladie des muqueuses qui constitue une sorte de cul-de-sac épidémiologique. D'autre part, comme les souches cytopathogènes sont incapables de se multiplier chez le foetus pendant la période critique de l'acquisition de la tolérance, elles ne peuvent pas générer des porteurs asymptomatiques immunotolérants de souches cytopathogènes. Les animaux infectés de manière persistante par des biotypes non cytopathogènes sont donc bien le réservoir de l'ensemble des souches des deux biotypes. Ces animaux sont en général peu nombreux dans les troupeaux $(38,39)$ mais sont responsables d'une circulation rapide du virus chez les animaux de même âge, ce qui explique le pourcentage élevé d'animaux porteurs d'anticorps dans les troupeaux (38). 


\section{CONCLUSION}

Les progrès réalisés ces dernières années dans la biologie moléculaire de l'infection par les Pestivirus ont abouti à une meilleure compréhension des mécanismes impliqués dans la pathogénie et l'épidémiologie de l'infection. Malgré ces progrès, certaines inconnues subsistent.

Il reste à éclaircir définitivement l'origine des souches responsables du déclenchement de la maladie des muqueuses : l'émergence d'un biotype cytopathogène à partir du biotype non cytopathogène persistant peut-elle s'expliquer par un seul mécanisme universel, ou résulte-t-elle tantôt d'une mutation, tantôt d'un phénomène de recombinaison dont les conséquences varieraient selon les souches?

L'autre inconnue est celle du rôle respectif des deux biotypes dans les affections néonatales du veau (19). La période néonatale semble de toute façon une période critique pour les animaux porteurs persistants du virus, qui souffrent notamment du fait de l'acquisition d'anticorps spécifiques d'origine maternelle par le biais du colostrum (7), provoquant l'accumulation d'immuncomplexes avec ses conséquences habituelles. Les mères n'étant généralement pas immunotolérantes préşentent en effet une réponse immune normale après leur infection primaire qui, chez les ruminants, n'interfère aucunement avec l'infection du fœetus. Les veaux infectés de façon persistante sont donc brusquement confrontés, après la naissance, à l'acquisition d'anticorps spécifiques d'origine maternelle. Une mortalité plus importante de ces animaux pourrait en résulter en période néonatale.

Enfin, il reste à démontrer que les caractéristiques épidémiologiques de l'infection par les Pestivirus chez les ruminants domestiques correspondent à un phénomène biologique général qui trouve son correspondant chez les ruminants sauvages auxquels il serait donc extrapolable. Il est par conséquent souhaitable d'étudier le mode de persistance des Pestivirus chez les ruminants sauvages, en particulier le cerf rouge chez qui l'infection a déjà été décrite.

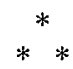

\section{ASYMPTOMATIC CARRIAGE OF PESTIVIRUSES BY RUMINANTS. - P.-P. Pastoret, D. Boulanger, B. Mignon and S. Waxweiler.}

Summary: Pestiviruses are enveloped single-chain ribonucleic acid viruses with a positive polarity. Pestiviruses include the viruses of classical swine fever (hog cholera), Border disease of sheep, mucosal disease of cattle, and isolates obtained from wild animals, such as red deer (Cervus elaphus). Among ruminants, pestiviruses have developed a remarkable strategy for assuring their persistence. Through epigenetic transmission, they lead to the birth of asymptomatic carrier animals harbouring non-cytopathic variants, which become immunotolerant to the strain of virus present.

The presence of a small number of asymptomatic carriers enables the virus to circulate within a herd by horizontal transmission, leading to the birth of a new generation of asymptomatic carriers.

KEYWORDS: Carrier state - Cervus elaphus - Epidemiology Immunotolerance - Pestivirus - Ruminants - Viral diseases. 


\title{
RUMIANTES PORTADORES ASINTOMÁTICOS DE PESTIVIRUS. - P.-P. Pastoret, D. Boulanger, B. Mignon y S. Waxweiler.
}

\begin{abstract}
Resumen: Los Pestivirus son virus con envoltura cuyo ácido ribonucleico de una sola cadena es de polaridad positiva. A este grupo pertenecen el virus responsable de la peste porcina clásica, el de la enfermedad de la frontera, el de la enfermedad de las mucosas y aislados obtenidos en mamíferos salvajes como el ciervo colorado (Cervus elaphus). Para asegurar su perennidad en los rumiantes, los Pestivirus han inventado una estrategia particularmente notable, que consiste en provocar el nacimiento, mediante transmisión epigenética, de animales portadores asintomáticos de variantes del virus exentas de citopatogenicidad e inmunotolerantes respecto de la cepa de virus que hospedan.
\end{abstract}

La presencia en los rebaños de un reducido número de portadores asintomáticos permite la circulación del virus por transmisión horizontal y garantiza así el nacimiento de una nueva generación de portadores asintomáticos.

PALABRAS CLAVE: Cervus elaphus - Enfermedades virales Epidemiología - Inmunotolerancia - Pestivirus - Porte - Rumiantes.

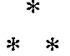

\section{BIBLIOGRAPHIE}

1. AKKINA R.K. (1991). - Pestivirus bovine viral diarrhea virus polypeptides: identification of new precursor proteins and alternative cleavage pathways. Virus Res., 19, 67-82.

2. Bielefeldt Ohmann H., Rønsholt L. \& Bloch B. (1987). - Demonstration of bovine viral diarrhoea virus in peripheral blood mononuclear cells of persistently infected clinically normal cattle. J. gen. Virol., 68, 1971-1982.

3. BoLIN S.R. (1988). - Viral and viral protein specificity of antibodies induced in cows persistently infected with non-cytopathic bovine viral diarrhea virus after vaccination with cytopathic bovine viral diarrhea virus. Am. J. vet. Res., 49, 40-44.

4. Bolin S.R., MCClurkin A.W., Cutlip R.C. \& CoRia M.F. (1985). - Severe clinical disease induced in cattle persistently infected with non-cytopathic bovine viral diarrhea virus by superinfection with cytopathic bovine viral diarrhea virus. Am. J. vet. Res., 46, 573-576.

5. BRownlie J. (1990). - Pathogenesis of mucosal disease and molecular aspects of bovine virus diarrhoea virus. Vet. Microbiol., 13, 371-382.

6. Brownlie J., Clarke M.C. \& Howard C.J. (1984). - Experimental production of fatal mucosal disease in cattle. Vet. Rec., 114, 535.

7. Brownlie J., Clarke M.C., Howard C.J. \& Pocock H. (1987). - Pathogenesis and epidemiology of bovine virus diarrhoea virus infection of cattle. In Meeting on Pestivirus. Liège, 8 avril 1986. Ann. Rech. vét., 18, 157-166.

8. Brownlie J., Clarke M.C. \& Howard C.J. (1989). - Experimental infection of cattle in early pregnancy with a cytopathic strain of bovine virus diarrhoea virus. Res. vet. Sci. 46, 307-311. 
9. Clarke M.C., Brownlie J. \& Howard C.J. (1987). - Isolation of cytopathic and non cytopathic bovine viral diarrhoea virus from tissues of infected animals. In Pestivirus infections of ruminants (J.W. Harkness, édit.). Commission des Communautés européennes, Rapport EUR 10238 EN, 3-10.

10. Collett M.S., Larson R., Gold C., Strick D., Anderson D.K. \& Purchio A.F. (1988). - Molecular cloning and nucleotide sequence of the pestivirus bovine viral diarrhea virus. Virology, 165, 191-199.

11. Collett M.S., Wiskerchen M., Welniak E. \& Belzer S.K. (1991). - Bovine viral diarrhea virus genomic organization. Arch. Virol. (suppl.), 3, 19-27.

12. CoraPi W.V., Donis R.O. \& Dubovi E.J. (1988). - Monoclonal antibody analysis of cytopathic and non cytopathic viruses from fatal bovine viral diarrhea virus infections. J. Virol., 62, 2823-2827.

13. Demeur C., Urbain J. \& Pastoret P.-P. (1990). - Immunotolérance. In Immunologie animale (P.-P. Pastoret, A. Govaerts \& H. Bazin, édit.). Flammarion, MédecineSciences, Paris, 163-168.

14. Demoerlooze L., Desport M., Renard A., Lecomte C., Brownlie J. \& Martial J.A. (1990). - The coding region for the 54-kDa protein of several pestiviruses lacks host insertions but reveals a "zinc finger-like" domain. Virology, 177, 812-815.

15. HAMBLin C. \& Hedger R.S. (1979). - The prevalence of antibodies to bovine viral diarrhoea/mucosal disease virus in African wildlife. Comp. Immun. Microbiol. infect. Dis., 2, 295.

16. Hewicker M., Wöhrmann T., Fernandez A., Trautwein G., Liess B. \& Moennig V. (1990). - Immunohistological detection of bovine viral diarrhoea virus antigen in the central nervous system of persistently infected cattle using monoclonal antibodies. $V e t$. Microbiol., 23, 203-210.

17. HorzineK M.C. (1991). - Pestiviruses - Taxonomic perspectives. Arch. Virol. (suppl.), 3, 1-5.

18. Howard C.J., Brownlie J. \& Clarke M.C. (1987). - Comparison by the neutralisation assay of pairs of non-cytopathogenic and cytopathogenic strains of bovine virus diarrhoea virus isolated from cases of mucosal disease. Vet. Microbiol., 13, 361-369.

19. Howard C.J., Clarke M.C. \& Brownlie J. (1989). - Protection against respiratory infection with bovine virus diarrhoea virus by passively acquired antibody. Vet. Microbiol., 19, 195-203.

20. Hughes L.E., Kershaw G.F. \& Shaw I.G. (1959). - "B" or Border disease. An undescribed disease of sheep. Vet. Rec., 71, 313-317.

21. KARSTAD L.H. (1981). - Bovine virus diarrhea. In Infectious diseases of wild animals ( 2 éd.). Iowa State University Press, 209.

22. Liess B., Frey H.-R., Kittsteiner H., Baumann F. \& NeumanN W. (1974). Beobachtungen und Untersuchungen über die «mucosal disease», des Rindes - einer immunbiologisch Erklärbaren Spätform der BVD-Virusinfektion mit Kriterien einer «slow virus infection»? Dt. tierärztl. Wschr., 81, 477-500.

23. MCClurkin A.W., Littledike E.T., Cutlip R.C., Frank G.H., Coria M.F. \& BoLIN S.R. (1984). - Production of cattle immunotolerant to bovine viral diarrhea virus. Can. J. comp. Med., 48, 156-161.

24. Malmouist W.A. (1968). - Bovine viral diarrhea-mucosal disease: etiology, pathogenesis and applied immunity. J. Am. Vet. Med. Assoc., 152, 763-768.

25. Meyers G., Rümenapf T. \& Thiel H.J. (1989). - Molecular cloning and nucleotide sequence of the genome of hog cholera virus. Virology, 171, 555-567.

26. Meyers G., Rümenapf T. \& Thiel H.J. (1989). - Ubiquitin in a togavirus. Nature, 341, 491. 
27. Meyers G., Tautz N., Dubovi E.J. \& Thiel H.J. (1991). - Viral cytopathogenicity correlated with integration of ubiquitin-coding sequences. Virology, 180, 602-616.

28. Moormann R.J.M., Warmerdam P.A.M., VAN DeR MeER B., SChaAper W.M.M., WENSVOORT G. \& HULST M.M. (1990). - Molecular cloning and nucleotide sequence of hog cholera virus strain Brescia and mapping of the genomic region encoding envelope protein E1. Virology, 177, 184-198.

29. Nettleton P.F., Barlow R.M., Gardiner A.C., Pastoret P.-P. \& Thiry E. (1985). - La pathogénie et l'épidémiologie de l'infection par le virus BVD. Ann. Méd. vét., 129, 93-108.

30. Olafson P., MCCallum A.D. \& FoX F.H. (1946). - An apparently new transmissible disease of cattle. Cornell Vet., 36, 205-213.

31. Pastoret P.-P., Thiry E. \& Dubuisson J. (1987). - Les porteurs de virus : analyse des états d'équilibre entre le virus et son hôte. In Meeting on Pestivirus, 8 avril 1986, Liège. Ann. Rech. vét., 18, 181-191.

32. Purchio A.F., Larson R., Torborg L.L. \& Collett M.S. (1984). - Cell-free translation of bovine viral diarrhea virus RNA. J. Virol., 52, 973-975.

33. Ramsey F:K. \& Chivers W.H. (1953). - Mucosal disease of cattle. Nth Amer. Vet., 34, 629-633.

34. Renard A., Guiot C., Schmetz D., Dagenais L., Pastoret P.-P., Dina D. \& MARTIAL J.A. (1985). - Molecular cloning of bovine viral diarrhea viral sequence. DNA, 4, 429-438.

35. Renard A., Schmetz D., Guiot C., Brown-Shimmer S., Dagenais L., Pastoret P.-P., DINA D. \& MARTIAL J.A. (1987). - Molecular cloning of the bovine viral diarrhea virus genomic RNA. In Meeting on Pestivirus, 8 avril 1986, Liège. Ann. Rech. vét., 18, 121-125.

36. Roeder P.L. \& Drew T.W. (1984). - Mucosal disease of cattle: a late sequel to fetal infection. Vet. Rec., 144, 309-313.

37. Steck F., Lazary S., Fey H., Wandeler A., Huggler C., Oppliger G., BAUMBERGER H., KADERKI R. \& MARTIG J. (1980). - Immune responsiveness in cattle fatally affected by bovine virus diarrhoea-mucosal disease. Zentbl. VetMed., 273, 429-445.

38. WaXweiler S., Karelle-Bui-Thi L., Sneyers M., Lambert A.F., Delchambre M., Dubuisson J., Thiry E., Antoine H., Dive M., Detal G. \& Pastoret P.-P. (1989). Circulation de souches non-cytopathogènes du virus BVD-MD dans des lots de taurillons. Ann. Méd. vét., 133, 681-690.

39. Waxweiler S., Karelle-Bui-Thi L., Boulanger D., Mignon B., Ernst E., Detal G., BOONEN F. \& PASTORET P.-P. (1992). - Bilan de cinq années de détection des taurillons infectés de manière persistante par le virus BVD au centre de sélection bovine de Ciney. Ann. Méd. vét., 136, 57-60.

40. Westaway E.G., Brinton M.A., Gaidamovich S. Ya., Horzinek M.C., Igarashi A., KÄÄRIÄInEN L., Lvov D.K., PORTERfield J.S., Russell P.K. \& TRENT D.W. (1985). Togaviridae. Intervirology, 24, 125-139.

41. Westaway E.G., Brinton M.A., Gaidamovich S. Ya., Horzinek M.C., Igarashi A., KÄÄriäIneN L., Lvov D.K., Porterfield J.S., RusSELl P.K. \& TRENT D.W. (1985). Flaviviridae. Intervirology, 24, 183-192.

42. Wiskerchen M., Belzer S.K. \& Collett M.S. (1991). - Pestivirus gene expression: the first protein product of the bovine viral diarrhea virus large open reading frame, p20, possesses proteolytic activity. J. Virol., 65, 4508-4514. 
43. WiskeRChEN M. \& ColLETT M.S. (1991). - Pestivirus gene expression: protein p80 of bovine viral diarrhea virus is a protein involved in polyprotein processing. Virology, 184, 341-350. 